\title{
Funcionamiento Ejecutivo en Adolescentes Embarazadas del Departamento de Sucre-Colombia: Una Respuesta Desde la Cognición.
}

\author{
Executive Functioning In Pregnant Adolescents In The \\ Department Of Sucre-Colombia: A Response From Cognition.
}

Katy Arroyo-Alvis, Andrés Ramírez-Giraldo, Janeth Salazar-López

\section{Resumen}

Introducción. El embarazo adolescente cambia el sentido de vida de las chicas y de sus familias, en ocasiones pueden ser producto de toma de decisiones desacertadas al momento de tener relaciones sexuales.

Objetivo: Describir el funcionamiento ejecutivo de adolescentes en estado de embarazo del departamento de Sucre.

Método: Enfoque cuantitativo, observacional, de un nivel descriptivo y de corte transversal; una muestra de 72 adolescentes, 36 de ellas en embarazo del departamento de Sucre, Colombia.

Muestra: método de selección y rechazo, aplicando un análisis de varianza simple, usando el programa R-Studio.

Instrumentos: se aplicó un protocolo de pruebas que contiene, Test de asociación controlado de palabras, Test de clasificación de Wisconsin (WCST), Test de Stroop, Test Del Trazo (Trail Making Test, TMT).

Resultados: Las adolescentes en embarazo presentaron mayor dificultad para tomar decisiones, fallas en la resolución de problemas, organización y planeación de la información, además pobre auto monitoreo, lento aprendizaje y poca velocidad de procesamiento de la información.

Conclusión: La maduración de áreas cerebrales con cambios ocurridos en la adolescencia son concordantes con las dificultades encontradas en este funcionamiento ejecutivo y la aparición de conductas de riesgo dando como resultado posibles embarazos durante la adolescencia.

Palabras clave: Función ejecutiva, adolescentes, embarazo temprano, conductas de riesgo

\section{Abstract}

Introduction. Teen pregnancy changes the way of life of girls and their families, sometimes they can be the product of poor decision-making when having sex.

Objective: To describe the executive functioning of adolescents in a state of pregnancy in the department of Sucre.

Method: Quantitative, observational, descriptive level and cross-sectional approach; a sample of 72 adolescents, 36 of them pregnant in the department of Sucre, Colombia.

Sample: selection and rejection method, applying a simple analysis of variance, using the R-Studio program.

Instruments: a test protocol was applied containing: Controlled word association test, Wisconsin classification test (WCST), Stroop test, Trail Making Test (TMT).

Results: Adolescent girls in pregnancy presented greater difficulty in making decisions, failures in problem solving, organization and planning of information, as well as poor self-monitoring, slow learning and low speed of information processing.

Conclusion: The maturation of brain areas with changes occurred in adolescence is consistent with the difficulties found in this executive functioning and the appearance of risk behaviors resulting in possible pregnancies during adolescence.

Keywords: Executive function, adolescents, early pregnancy, risk behavior 


\section{Introducción}

Desde cualquier esfera o punto de partida de estudio, la adolescencia es una etapa crucial en la vida de todo ser humano. Desde las neurociencias, esta es una etapa vital en la cual el sujeto se encuentra sumergido en las constantes conexiones sinápticas, mismas que indican que aún no ha alcanzado su madurez, sino que está en pro de su desarrollo cerebral. Es decir, es un modo de trabajo inacabado o en progreso; ${ }^{1}$ dichas conexiones constituyen entre los componentes más importantes para que el desarrollo infantil y adolescente sea exitoso?

El adolescente no ha alcanzado su culmen cerebral madurativo, en esta etapa la corteza frontal se ve sometida ante constructos tales como inhibición de impulsos, toma de decisiones, flexibilidad; las cuales, se hacen evidente a través de estudios de imagen cerebral como lo son, la Resonancia Magnética Funcional (IRMF) y la Tomografía de Emisión de Positrones (PET); ${ }^{3}$ es por ello que, algunos autores haciendo un comparativo entre sujetos adultos con lesiones en los lóbulos frontales y adolescentes, notan la presencia de cambios conductuales y cognitivos en estos primeros, que van desde dificultades en el control inhibición de los impulsos, dificultades para sostener la atención y problemas en la memoria a corto plazo, también presentan incapacidad para omitir información poco relevante, trayendo como consecuencia la alta distracción y el pobre desempeño en tareas sencillas que requieran los procesos anteriormente mencionados; por concerniente estiman en concluir que la similitud de cognición y comportamientos que tienen estos adultos frontalizados con los adolescentes se basan en la falta de maduración o lesión en dichos circuitos. ${ }^{4-6}$

Por otro lado, si bien históricamente se ha vinculado al funcionamiento de esta corteza prefrontal con las llamadas Funciones ejecutivas (FE); éstas, también se han llamado "funciones frontales." En sinónimo de la conjunción y cuyo periodo es crítico en los adolescentes (CIATA), también existen autores quienes no respaldan la relación uno a uno entre las funciones ejecutivas y la actividad del lóbulo frontal $;^{7}$ parten de la premisa que en la adolescencia ocurren picos de desarrollo relacionados al funcionamiento frontal, en sus subfunciones y en sus alteraciones; todas ellas residirán con fenómenos que sobrevienen en la adolescencia con por ejemplo embarazo temprano.

Ahora bien, el desarrollo ejecutivo desde la infancia hasta la edad de madurez cognitiva, siempre ha sido un plano generosamente estudiado y de especial interés para los psicólogos del desarrollo; donde la memoria de trabajo, el control inhibitorio y la flexibilidad cognitiva, son componentes céntricas que constituyen el conjunto de estos procesos denominados FE y que a través de estudios de neuroimagen siguen sumiendo su base en ese funcionamiento frontal. ${ }^{3}$ En esta línea de ideas, con respecto a la adolescencia diversos autores convergen en sostener que paralelo al proceso de maduración cerebral, también se hace importante tener en cuenta otros factores como lo son los cambios físicos y psicológicos, así como, el contexto sociocultural del adolescente. Con estos, se logra dar una explicación a su forma de pensamiento o de procesamiento especialmente en lo concerniente a los procesos o habilidades tales como, toma de decisiones, memoria de trabajo, control inhibitorio, control atencional, control consciente del pensamiento, afectividad y comportamiento, lo cual precisamente se engloba en la categoría de las FE, éstas son predictores de las competencias sociales y el rendimiento académico ${ }^{1,5,4,7,8}$ y de vital importancia para una adaptación exitosa que permea comportamientos dirigidos y la capacidad de manejar múltiples demandas de desempeño en varias esferas del sujeto.

Es por ello que investigaciones sugieren que los adolescentes son quienes presentan mayor riesgo de presentar comportamientos en busca de gratificaciones a corto plazo (uso de sustancias, malos hábitos alimenticios, abandono escolar $u$ otros comportamientos peligrosos, o poco responsables como relaciones sexuales sin protección), a pesar del daño potencial y a costa de los resultados a largo plazo. ${ }^{10}$ Esto, va de la mano con funciones subyacentes de orden superior como razonamiento, resolución de problemas y planificación, ${ }^{11}$ que desde un punto de vista neuropsicológico son quienes representan ese sistema de regulación y control de los procesos psicológicos y cuya base sigue apoyándose en

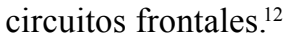

Con respecto a los cambios orgánicos presentados en la etapa evolutiva de la adolescencia, las modificaciones físicas más notables, son las relacionadas a los órganos sexuales masculinos y femeninos, además de la aparición de los períodos menstruales en las adolescentes, y la maduración consecuente de la figura anatómica de ambos géneros. Adherido a esto, el grupo etario en mención atraviesa en conjunto, diferencias psicológicas que les hace abordar su paso de la infancia a la adolescencia; es decir, cada sujeto enfrenta el proceso de dejar de ser niño, para convertirse en un sujeto consciente del poder de tomar decisiones propio de esta etapa. A grandes rasgos el hecho de dejar de ser niños, trae consigo un sin número de afectaciones emocionales, puesto que le exige al sujeto enfrentarse ante la responsabilidad de decidir, que implica mínimamente tener claras las opciones entre las que elige, para así, hacer su mejor esfuerzo para apuntar hacia lo que mejor sea conveniente. Esto puede generar en el menor, estrés, miedos, frustraciones, entre otros inconvenientes emocionales; ${ }^{13-15}$ otros autores estiman que es justamente en la adolescencia donde las demandas de autonomía y autorregulación aumentan. ${ }^{16}$ 
Por otro lado, hay autores ${ }^{13,15}$ que estiman varias facetas en las que un joven atraviesa y que lleva implícito ese instinto de desarrollo sexual garante de su desarrollo, y en teoría, un equilibrio psicológico en todo ese proceso. En ese sentido un primer periodo inicial que tiene lugar en edades comprendidas entre 9-11 años, en este comienzan a tener de manera más tangible los cambios anatómicos y a nivel psicológico, se denotan cambios emocionales tanto en niños como en niñas. A nivel masculino, se les percibe ciertos comportamientos agresivos hacia las féminas; y con respecto a las chicas se percibe en ellas la proliferación de rasgos y destrezas masculinas. Seguidamente, se vivencia una segunda fase en edades entre 12-14 años; sus principales rasgos psicológicos característicos en los adolescentes se evidencian en la lejanía que van tomando de sus padres y la generación de nuevos vínculos sociales, sin dejar de lado el interés siempre presente, por la genitalidad y consecuentemente el estímulo de la sexualidad. La tercera etapa se ubica entre los 16-18 años, se da lugar con mayor firmeza la identificación o elección de ser heterosexual, homosexual o bisexual; acompañado por procesos conjuntos donde comienza a enfrentar la consecuente duda existencial sobre su propósito vital y enfrenta procesos de duelo, al alejarse de las figuras parentales, y enamoramiento al dar inicio con relaciones amorosas. Consecuentemente una cuarta fase se presenta entre los 19 y 21 años, en esta, se tiene como prioridad la consolidación de criterios para sentar bases sólidas con respecto a la visión de la vida y las consecuentes decisiones que se toman en pro de ella. Y finalmente se da una quinta etapa entre los 21-24 años, en esta, se inicia la madurez psicológica y se reafirman procesos de individualización, adopción de conductas socialmente aceptables, entre otros factores importantes para la preparación de la vida adulta.

En consecuencia con los cambios que acarrea la adolescencia, se da inicio a la actividad sexual, estimándose que los adolescentes comienzan las prácticas sexuales entre los 14 y 16 años, esto se acuña, no solo al hecho de los cambios físicos, sino también a factores cognitivos propios de la edad, por los cuales los jóvenes guardan un sentido de osadía y no se percatan de los riesgos asumidos; en otras palabras, los adolescentes creen que las decisiones que toman no tendrán consecuencias negativas, tienen cierto sentimiento de invulnerabilidad y en

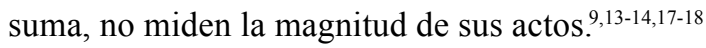

La adolescencia regularmente es caracterizada por perseverancias de conductas de riesgo y dentro de ellas es notoria las drogas o el sexo sin protección; ${ }^{16}$ hay autores que respaldan estas conductas de osadía, con el incompleto desarrollo de las funciones efectivas y más especialmente con la incorporación del control emocional, conducta moral y desarrollo del juicio a la esfera ejecutiva. ${ }^{19}$
En esta línea de ideas, otros autore ${ }^{20}$ mencionan que la sexualidad y reproducción adolescente se trata como un problema de alto impacto, no solo en lo que incumbe en el área de la medicina, donde se generan altos índices de mortalidad, sino también a los aspectos psicológicos y socioculturales; produciendo así, el detrimento la calidad de vida, el abandono de las actividades escolares y otros aspectos de tipo económico que determinan el desarrollo de una región o país. En otras palabras, a mención de estos autores, el embarazo adolescente o embarazo precoz, constituye una problemática de salud pública; ${ }^{15,20-21}$ afectando primordialmente a los jóvenes de países que se encuentran en vía de desarrollo. Cada año se presenta el nacimiento aproximado de un millón de neonatos cuyas madres son menores de 15 años, además anualmente se presentan dieciséis millones de casos de adolescentes embarazadas ubicadas en un grupo etario de 15 a 19 años, y estas en su mayoría son originarias de países en desarrollo. A esto se suma, que la tasa de mortalidad neonatal es mucho mayor en los bebes cuyas progenitoras tienen entre 14-19 años, que aquellos neonatos que son hijos de mujeres con edades entre 20-24 años. ${ }^{22}$

Ahora bien, el abordaje del embarazo en adolescentes y todo lo que sobreviene del neurodesarrollo de funciones, como lo son las FE, han sido poco estudiadas. Se encontraron estudios que dan respuesta desde lo social, familiar, educativo y cultural; pero estudios que integran este patrón de respuesta en la línea de caracterización desde la aproximación biológica son escasos; y si a eso agregamos que aunque a nivel de literatura investigativa en la adolescencia ya se cuenta con decenas de artículos sobre esta temática, recientes trabajos de revisión y metanálisis advierten que aún no se ha conseguido construir un conocimiento básico sobre el desarrollo infancia-adolescencia, que explique y ayude a la prevención de conductas de riesgo acontecidas en esta etapa como lo es un embarazo a temprana edad..$^{23}$

El presente estudio pretende levantar una línea base de funcionamiento ejecutivo en adolescentes embarazadas del departamento de Sucre, con miras de detallar el funcionamiento y/o alteraciones en estos procesos de crucial importancia en la regulación psicológica y biológica, ${ }^{12}$ que acarrea esta etapa del desarrollo en aras de aproximar una línea biológica de explicación.

\section{Método}

Este estudio se enmarca en el ámbito de un paradigma positivista, de corte transversal, de tipo observacional con nivel descriptivo-comparativo. La muestra fue intencional, conformada por un grupo de 36 adolescentes embrazadas del departamento de Sucre y un grupo control de 36 mujeres jóvenes que no han presentado embarazo hasta el momento. Los grupos se construyeron en 4 
municipios de este departamento (Buenavista, corozal y Morroa). Las edades oscilaban de 14 a 20 años. Los instrumentos empleados fueron: Test de asociación controlado de palabras, Test de clasificación de Wisconsin (WCST), Test de Stroop, Test Del Trazo (Trail Making Test, TMT), que buscaban medir el funcionamiento ejecutivo en ámbitos cognitivos, comportamentales y emocionales.

\section{Procedimiento}

En una primera fase se realizó visita a las secretarias de salud de los municipios de Sincé, Buenavista, Corozal, Morroa y Sincelejo del departamento de Sucre, con el fin de presentar el proyecto y su intencionalidad, una vez se contó con el aval y participación, se accedió a las bases de datos de las mujeres embarazadas en cada municipio, ubicando únicamente las embarazadas en un grupo etario entre 14-19 años. Se procedió a contactar a cada una de las adolescentes embarazadas, con el fin de proceder a la firma del consentimiento informado de las mismas y de los adultos a cargo de ellas, esto debido a que son menores de edad; adicionalmente, se da inicio a la aplicación del protocolo neuropsicológico por medio del cual se evalúa el estado ejecutivo de cada menor.

\section{Diseño experimental}

Para la selección de la muestra se aplicó el método de selección y rechazo, el cual es un algoritmo de selección que se desarrolla mediante la aplicación de los pasos que permite equiparar muestras semejantes; para el análisis estadístico de los datos se realizó un diseño experimental bajo arreglo completamente al azar (DCA), donde el factor que se consideró como fuente de variación provienen de los grupos de estudio y la variación de error que se produce por es una medida de la variación existente entre las observaciones medidas sobre unidades experimentales tratadas en forma similar. Para tal propósito, se aplicó un análisis de varianza simple o de un solo factor, cuyo propósito fue probar las hipótesis apropiadas en torno a la media de los grupos y estimarlas. En el presente estudio, se desea determinar si existe diferencia en los valores medios de las variables de cada test que evalúan componentes de las funciones ejecutivas en función de los grupos de estudio y el control. Para determinar si existe diferencia para las variables de respuesta en función de los grupos de estudio, se considera el siguiente juego de hipótesis:

\section{Hipótesis nula}

$$
H_{1}: \mu 1=\mu 2=\mu 3=\mu 4=\mu
$$

Los valores medios de las variables de respuesta del estudio son iguales para cada uno de grupos es estudio.
Para comprobar la hipótesis nula, se realizó un análisis de varianzas empleando el programa R-Studio bajo licencia GNU. Para las variables de respuesta que arrojaron diferencias significativas entre sus valores medios se aplicó la prueba de comparaciones múltiples de Tukey a un nivel de significancia del 5\%.

\section{Resultados}

Dentro de los resultados (Tabla 1), se encontraron diferencias entre los grupos focos de análisis (adolescentes embarazadas/grupo control), viéndose fallas de tipo cognitivo y comportamental; notándose puntajes con significancia en WCST: Aciertos (P .000), errores (P .000), categorías (P .000), total de ensayos (P .009), errores no perseverativos (P .000), índice de conceptualización inicial (P .000), respuesta de nivel conceptual (P .000), porcentaje de conceptualización (P .000), otros (P .012), TMT B tiempo (P .000).

Tabla 1. Resultados de análisis de varianza.

\begin{tabular}{|cc|}
\hline Variables de funcionamiento ejecutivo & Valor $\mathbf{P}$ \\
\hline WCST Aciertos & $.000912^{* * *}$ \\
\hline WCST Errores & $.000476^{* * *}$ \\
\hline WCST Categorías & $.000184^{* * *}$ \\
\hline WCST Total Ensayos & $.00962^{* *}$ \\
\hline WCST Respuestas perseverativas & $.482^{*}$ \\
\hline WCST Errores no perseverativos & $.000653^{* * *}$ \\
\hline WCST Errores perseverativos & $.129^{*}$ \\
\hline WCST Porcentaje de erores no perseverativos & .0901 \\
\hline WCST índice de Conceptualización inicial & $.000318^{* * *}$ \\
\hline WCST Respuestas del nivel conceptual & $.000104^{* * *}$ \\
\hline WCST Porcentaje de conceptualización & $2.01 \mathrm{e}-05^{* * *}$ \\
\hline WCST Fallas para mantener el principio & .697 \\
\hline WCST Otro & $.0127^{*}$ \\
\hline TMT B tiempo & $3.15 \mathrm{e}-06^{* * *}$ \\
\hline TMT errores & $.124^{*}$ \\
\hline FAS total & .989 \\
\hline Stroop conflicto & \\
\hline
\end{tabular}

Con base a los resultados (Tabla 1), se puede encontrar que estas adolescentes embarazadas del departamento de Sucre, presentan mayor riesgo para tomar decisiones frente al acto sexual, dificultando el análisis del

\section{Hipótesis alternativa}

\section{$H_{1}: \mu i \neq \mu j$ paraalgun $i \neq j$}

Los valores medios de las variables de respuesta del estudio no son iguales en al menos un par de tratamientos para cada uno de grupos es estudio. 
mismo de manera cognitiva y conductualmente; esto podría estar mediado por fallas para la resolución de problemas, organización y planeación de la información; basando su conducta en errores debido al alzar (WCST - Aciertos/categorías); sumado a eso, la carga hormonal típica de la etapa de desarrollo es concordante con la presencia de respuesta prepotente incorrecta, fallas en control inhibitorio, y pobre automonitoreo (WCST - errores/ errores no perseverativos); acompañado de lento aprendizaje y poca velocidad de procesamiento de la información que repercute en una ineficaz respuesta frente situaciones ambientales o externas (WCST- conceptualización inicial/Respuesta del nivel conceptual y porcentaje del nivel conceptual).

En el análisis de comparaciones (Tabla 2) se encontró menor rendimiento en la gran mayoría de las pruebas de funcionamiento ejecutivo, notándose menor cantidad de aciertos en WCST, en relación a grupo caso; y en consecuencia menor número de categorías, necesitando mayor número de ensayos para lograr de forma categórica las demandas, dificultando el cambio de estrategias cognitivas, formación de conceptos y memoria de trabajo.

Tabla 2. Resultados de comparaciones múltiples de FE entre los grupos.

\begin{tabular}{|lcc|}
\hline $\begin{array}{l}\text { Variables de } \\
\text { Funcionamiento Ejecutivo }\end{array}$ & $\begin{array}{c}\text { Grupo } \\
\text { Caso }\end{array}$ & $\begin{array}{c}\text { Grupo } \\
\text { Control }\end{array}$ \\
\hline WCST Aciertos & $61.14^{\mathrm{b}}$ & $74.17 \mathrm{a}$ \\
\hline WCST Errores & $63.11^{\mathrm{a}}$ & $44.60 \mathrm{~b}$ \\
\hline WCST Categorías & $2.74^{\mathrm{b}}$ & $4.45 \mathrm{a}$ \\
\hline WCST Total Ensayos & $126.40^{\mathrm{a}}$ & $118.68 \mathrm{~b}$ \\
\hline WCST Errores no perseverativos & $32.80^{\mathrm{a}}$ & $19.80 \mathrm{~b}$ \\
\hline WCST índice de Conceptualización inicial & $53.57^{\mathrm{a}}$ & $20.97 \mathrm{~b}$ \\
\hline WCST Respuestas del nivel conceptual & $42.54^{\mathrm{b}}$ & $61.97 \mathrm{a}$ \\
\hline WCST Porcentaje de conceptualización & $33.00^{\mathrm{b}}$ & $53.57 \mathrm{a}$ \\
\hline WCST Otro & $2.34^{\mathrm{a}}$ & $0.94 \mathrm{~b}$ \\
\hline TMT B tiempo & $194.00^{\mathrm{a}}$ & $87.45 \mathrm{~b}$ \\
\hline $\begin{array}{l}{ }^{*} \text { Prueba de comparaciones múltiples de Tukey } \\
\text { **a=valor medio }\end{array}$ & \\
\hline
\end{tabular}

Se evidencia igualmente que estas jóvenes presentan mayor número en el total de errores y errores sin perseverancia, notándose reincidencia en la conducta corregida, manifestando así lento aprendizaje en la tarea.

Finalmente, todo lo anterior se sumen en fallas en el ejecutivo central y con él, pobre auto monitoreo, velocidad de procesamiento y construcción cognitiva que se aprecian en el pobre rendimiento en las tareas de conceptualización y TMT B tiempo; en los cuales el mayor valor promedio se inclinó hacia el grupo control. El rendimiento en el test de FAS, no marcó diferencia de importancia en rendimiento, ni significancia entre los grupos.

\section{Discusión}

Con respecto a las variables de discusión es pertinente mencionar que gran parte de este funcionamiento ejecutivo suele vincularse al funcionamiento frontal y más especialmente a la corteza pre frontal. Con ello diversos estudios afirman en la línea de maduración y desarrollo de esos circuitos y su conexión con otras regiones subcorticales como la amígdala, suelen iniciar su maduración desde temprano en la infancia y continúan incluso hasta el final de la adolescencia y todo esto es un interjuego y maduración en paralelo con la corteza prefrontal:;4-25 de manera que es posible contrastar gran parte de los resultados dados en la presente investigación relacionadas con estas dificultades ejecutivas en estos adolescentes desde la falta de maduración presente en estos circuitos corticorticales y cortico-límbicos.

Seguidamente, se ha manifestado dentro de los resultados la conducta impulsiva, puesta en evidencia en las fallas presentes de estas jóvenes hacia la perseverancia y el pobre auto monitoreo, costándole el análisis cognitivo de la conducta y sus consecuencias a largo plazo. Ahora bien, este tipo de conductas son usualmente vistas a esta edad y desde la línea biológica, ${ }^{16}$ explican que esta incidencia en conductas de riesgo obedece a la misma zaga de maduración que no solo implica el desarrollo de funciones ejecutivas de tipo cognitivas puras llamadas frías, sino también las de tipo emocional llamadas por su parte calientes ${ }^{26}$ y estas a su vez con circuitos de recompensa hallados en la misma corteza prefrontal. Lo anterior, es también respaldado por $\mathrm{Crone}^{27}$ quien describe que esta activación rápida de circuitos de recompensa y pobre de los circuitos prefrontales de autorregulación podrían contribuir a estas conductas de riesgo durante la adolescencia.

Ambos sistemas de grupos cognitivos (Frios) y emocionales (Calientes) integran una red neuronal fundamental para la regulación de la conducta; y el equilibrio entre estos dos subsistemas de pensamiento y emoción es crítico para la capacidad del individuo de generar respuestas reflexivas ${ }^{28}$ Es esto último lo que respondería al porqué del lento aprendizaje en estas jóvenes del departamento de Sucre y la pobre toma de decisiones positivas al momento de valorar una situación como es el sexo sin protección; adoptando por su parte una respuesta poco reflexiva que llevaría a tomar decisiones basados en la emoción y el momento, sin medir las consecuencias de esa conducta a largo plazo.

Algunos autores ${ }^{28}$ asocian este tipo de comportamiento como un procesamiento automático; y en consecuencia $^{16}$ le coloca una carga emocional alta que representa en el adolescente respuestas personales significativas, lo que los llevaría a añadir mayor dificultad para generar reflexiones frente a cualquier situación y más las de este tipo de conductas de riesgo. 
Por otro lado, otros autores ${ }^{19}$ en concordancia con la línea de desarrollo ejecutivo y maduración de circuitos prefrontales en la adolescencia, introducen aspectos como control emocional, conducta moral y desarrollo del juicio; los cuales son bastante bajos en este grupos de adolescentes; donde existe una pobre connotación de la conducta sexual, el juicio basado en la emoción y no en la cognición y finalmente lo que esto implica como decisión; a lo cual también se podría relacionar igualmente con el incompleto desarrollo de las mismas funciones ejecutivas ${ }^{29}$

Por otro lado, el aspecto socioeconómico se considera un factor que afecta al desarrollo tanto físico como cognitivo; ${ }^{16}$ y este último regularmente se expresa en pruebas de rendimiento académico e intelectuales en donde esa desigualdad social inclina la balanza en aquellos de estratos más bajos ${ }^{30-31} \mathrm{En}$ concordancia con lo anterior, es de notar que la población en cuestión representa una muestra del departamento de Sucre con ingresos económicos bajos y limitados; con lo cual las condiciones de vivienda, vecindario, planteles educativos e incluso factores de estimulación medioambientales como bibliotecas, acceso a internet, parques, se muestran escasos. Entonces, se podría hipotetizar que indirectamente este factor socioeconómico también puede estar asociado en el desarrollo óptimo a nivel cognitivo e incluso ejecutivo en estas jóvenes. Finalmente, lo anterior descrito es soportado por Pérez, et al. ${ }^{16}$ quienes concluyen en su escrito, que la condición socioeconómica repercute de forma negativa sobre el desarrollo y maduración de las Funciones Ejecutivas.

En conclusión, la presente investigación se llevó acabo para la identificación del funcionamiento ejecutivo en un grupo de adolescentes en embarazo del departamento de Sucre, explicando desde una línea neurocognitiva la relación entre el desenvolvimiento de las Funciones Ejecutivas y la condición de embarazo temprano. En concordancia con lo planteado se detalla en primera instancia que estas jóvenes presentan fallas más notorias en componente de orden cognitivo en relación a lo comportamental; encontrando que más que la conducta sexual impulsiva; esta responde a un orden hormonal inmerso en la misma conducta de riesgo típica del adolescente y que más bien la falla más importante reside en la valoración cognitiva del mismo acto; manifiesta en conductas poco reflexivas.

Así mismo, se encontró un significativo lento aprendizaje, seguido de reducción en la velocidad del procesamiento; lo que implicaría que aparte de las dificultades de análisis cognitivo, en la planeación y en su capacidad de automonitoreo conductual; estas chicas tardaron más en idear el desarrollo de estrategias cognitivas adecuadas, lo que podría llevarlas a una respuesta de condicionamiento automático mediada por la gratificación y recompensa del momento, en este caso, tener sexo sin pensar en las consecuencias futuras.
Por otro lado, no se encontraron dificultades en la conciencia fonológica ni en su memoria de trabajo, por lo que estas adolescentes conservan las habilidades de reconocimiento y búsqueda cognitiva de significados y connotaciones cuando están involucradas en un preámbulo sexual; pero aun así, son llevadas por conductas que busquen activaciones de centros de recompensa.

A nivel general, se puede concluir que la conducta sexual en el adolescente y más específicamente lo que implicaría un embarazo temprano, se podría asociar con la pobre maduración y estimulación del funcionamiento ejecutivo; encontrando principalmente funcionamiento deficiente en procesos de orden cognitivo como resolución de problemas, automonitoreo, planeación, desarrollo de estrategias cognitivas positivas, aprendizaje y procesamiento de la información. Todo lo anterior representa una línea biológica de interpretación desde el activo funcionamiento de áreas prefrontales de orden conductual y pobre activación en áreas cognitivas, lo que implica un funcionamiento frontal típico en el adolescente; además, sumado a ello se agrega la connotación del bajo estrato socioeconómico que permea la baja estimulación medioambiental que no favorece al óptimo desarrollo en estas funciones.

\section{Referencias}

1. Weinberger DR, Elvevåg B, Giedd, JN. The adolescent brain. Washington, DC: National Campaign to Prevent Teen Pregnancy. 2005. https://mdcune.psych.ucla.edu/ ncamp/files-fmri/NCamp_fMRI_AdolescentBrain.pdf

2. Diamond A, Lee K. Interventions shown to Aid Executive Function Development in Children 4-12 Years Old. Science, 2011; 333(6045): 959-964. https://doi. org/10.1126/science.1204529

3. Fiske A, Holmboe K. Neural substrates of early executive function development, Developmental Review. 2019; 52: 42-62; ISSN 0273-2297. https://doi. org/10.1016/j.dr.2019.100866

4. Delgado AO. Desarrollo cerebral y asunción de riesgos durante la adolescencia. Apuntes de psicología. 2007; 25(3): 239-254. https://psicopedia.org/wp-content/ uploads/2014/06/Riesgos-en-la-adolescencia.pdf

5. Stelzer F, Cervigni MA, Martino P. Bases neurales del desarrollo de las funciones ejecutivas durante la infancia y adolescencia. Una revisión. Revista chilena de Neuropsicología. 2010; 5(3): 176-184. https:// www.redalyc.org/pdf/1793/179318868001.pdf

6. Stelzer F, Cervigni MA. Desempeño académico y funciones ejecutivas en infancia y adolescencia. Una revisión de la literatura. Revista de investigación en educación. 2011; 9(1): 148-156. http://reined.webs. uvigo.es/index.php/reined/article/view/107

7. Oliva A, Antolín L. Cambios en el cerebro adolescente y conductas agresivas y de asunción de riesgos. Estudios de psicología. 2010; 31(1): 53-66. https://doi. org/10.1174/021093910790744563 
8. Becerra-Lagos D, Lepe-Martínez, N, Ramos-Galarza. Las funciones ejecutivas del lóbulo frontal y su asociación con el desempeño académico de estudiantes de nivel superior. Revista ecuatoriana de neurología. 2018; 27 (3): 51-56. http://revecuatneurol.com/wp-content/ uploads/2019/04/2631-2581-rneuro-27-03-00051.pdf

9. Álvarez J, Emory E. (2006). Executive function and the frontal lobes: A meta-analytic review. Neuropsychology review. 2006; 16 (1): 7-42. https://doi. org/10.1007/s11065-006-9002-X.

10. Noël X, Brevers D, Bechara A. Un enfoque neurocognitivo para comprender la neurobiología de la adicción. Current Opinion in Neurobiology. 2013; 23(4): 632 - 638. https://doi.org/10.1016/j.conb.2013.10.01.

11. Diamond A. Funciones ejecutivas. Informe annual de psicología. 2013; 64: 135-168. https://doi.org/10.1146/ annurey-psych-113011-143750.

12. Luria A. Las funciones corticales superiores del hombre. México: Fontamara. 1986.

13. Morillo J, Montero L. Lactancia materna y relación materno filial en madres adolescentes. Enfermería Global. 2010; 9(2): 1 de 9. http:// scielo.isciii.es/scielo.php?script=sci_arttext\&p id $=$ S1695-61412010000200019

14. Trujillo EV, Henao J, González C. Toma de decisiones sexuales y reproductivas en la adolescencia. Acta colombiana de psicología. 2007; 10(1): 49-63. https://psycnet.apa.org/record/2007-14928-005

15. Narváez-Rodríguez PJ. Perfil psicológico y social de las adolescentes en edades entre los 15 a 19 años que presentan embarazo precoz (Bachelor's thesis). Quito: UCE. 2017. http://www.dspace.uce.edu.ec/ handle/25000/12862

16. Pérez E, Carboni A, Capilla A. Desarrollo anatómico y funcional de la corteza prefrontal. En: Tirapu J, García A, Ríos M, Ardila A (Eds.), Neuropsicología de la corteza prefrontal y las funciones ejecutivas (pp. 175---195). Barcelona: Viguera. 2012.

17. González-Galbán H. (2000). Aspectos teóricos para el estudio sociodemográfico del embarazo adolescente. Frontera norte. 2000; 12(23): 65-85. https:// www.redalyc.org/pdf/136/13602303.pdf

18. BurunatE. El desarrollo del sustrato neurobiológico de la motivación y emoción en la adolescencia:¿ un nuevo período crítico?. Infancia y aprendizaje. 2004; 27(1): 87-104. https://doi.org/10.1174/021037004772902123.

19. Welsh MC, Pennington BF, Groisser DB. A normative developmental study of executive function: A window on prefrontal function in children. Developmental Neuropsychology. 1991; 7(2). 131-149. https:// doi.org/10.1080/87565649109540483.

20. Molina M, Ferrada C, Pérez R, Cid L, Casanueva V, García A. Embarazo en la adolescencia y su relación con la deserción escolar. Revista Médica de Chile. 2004; 132: 65-70. http://dx.doi.org/10.4067/S003498872004000100010 .
21. Moreno, S., Canelón, M. \& Becerra, L. (2006). Conducta sexual, conocimiento sobre embarazo y necesidades percibidas con relación a educación sexual, en adolescentes escolarizados. Espacio abierto. 2006. 15(4): 787-803. https://www.redalyc.org/ pdf/122/12215405.pdf

22. OMS. El embarazo en la adolescencia. 2020. https:// www.who.int/es/news-room/fact-sheets/detail/adolescent-pregnancy

23. Flores J, Castillo R, Jiménez N. Desarrollo de funciones ejecutivas, de la niñez a la juventud, Anales de psicología. 2014; 30(2): 463-473. https://doi. org/10.6018/analesps.30.2.155471.

24. Lewis MD, Lamm C, Segalowitz SJ, Stieben J, Zelazo PD. Neurophysiological Correlates of Emotion Regulation in Children and Adolescents. Journal of cognitive neuroscience. 2006; 18(3): 430-443. https://doi. org/10.1162/jocn.2006.18.3.430

25. Liston C, Watts R, Tottenham N, Davidson MC, Niogi S, Ulug AM, Casey BJ. Frontostriatal Microstructure Modulates Efficient Recruitment of Cognitive Control, Cerebral Cortex. 2006; 16(4): 553 -560. https://doi.org/10.1093/cercor/bhj003

26. Zelazo PD, Müller U, Frye D, Marcovitch S, Argitis G, Boseovski J. The development of executive function in early childhood. Monographs of the Society for Research in Child Development.2003; 68(3): 123-137. https://doi.org/10.1111/j.0037-976x.2003.00260.x.

27. Crone EA. Executive functions in adolescence: inferences from brain and behavior. Developmental science review. 2009; 12(6): 825 -830. https://doi. org/10.1111/j.1467-7687.2009.00918.x

28. Cunningham, WA, Zelazo PD. Attitudes and evaluations: a social cognitive neuroscience perspective. Trendsin Cognitive Sciences. 2007; 11(3): 97-104. https://doi.org/10.1016/j.tics.2006.12.005

29. Craun E, Lachance K, Williams C, Wong MM. Parent depressive symptoms and offspring executive functioning, Journal of Clinical and Experimental Neuropsychology. 2009; 41(2): 147-157. https://doi.org /10.1080/13803395.2018.1504893.

30. Hackman D, Farah M, Meaney M. (2010). Socioeconomic status and the brain: mechanistic insights from human and animal research. Nat Rev Neurosci. 2010; 11: 651-659. https://doi.org/10.1038/nrn2897

31. Vikram K, Chen F, Desai S. (2018). Mothers' work patterns and Children's cognitive achievement: Evidence from the India Human Development survey. Social Science Research. 2018; 72: 207-224. https:// doi.org/10.1016/j.ssresearch.2018.02.003.

Financiamiento: Lo realiza la Corporación Universitaria del Caribe en su convocatoria de proyectos internos de investigación 2018.

Conflicto de intereses: Los autores no presentan ningún conflicto de intereses. 